Proyecciones Journal of Mathematics

Vol. 33, $\mathrm{N}^{\circ}$ 4, pp. 415-435, December 2014.

Universidad Católica del Norte

Antofagasta - Chile

\title{
Fréchet differentiation between Menger probabilistic normed spaces
}

\author{
N. Eghbali \\ University of Mohaghegh Ardabili, Iran \\ Received: May 2014. Accepted: August 2014
}

\begin{abstract}
In this paper, we define and study Menger weakly and strongly Pconvergent sequences and then Menger probabilistic continuity. We also display Fréchet differentiation of nonlinear operators between Menger probabilistic normed spaces.
\end{abstract}

Subjclass : $46 S 40$.

Keywords : Menger probabilistic normed spaces; Fréhet differentiation; nonlinear operators. 


\section{Introduction}

The notion of probabilistic metric spaces was introduced by Menger [7]. Menger proposed transferring the probabilistic notions of quantum mechanic from physics to the underlying geometry. Probabilistic normed spaces are real linear spaces in which the norm of each vector is an appropriate probability distribution function rather than a number. The theory of probabilistic normed spaces was introduced by Š erstnev in 1963 [9]. In [1] Alsina, Schweizer and Sklar gave a new definition of probabilistic normed spaces which includes $\breve{S}$ erstnev's as a special case and leads naturally to the identification of the principle class of probabilistic normed spaces, the Menger spaces.

The idea of Menger was to use distribution function instead of nonnegative real numbers as values of the metric. It corresponds to the situation when we do not know exactly the distance between two points, we know only probabilities of possible values of this distance. The probabilistic generalization of metric spaces appears to be well adapted for the investigation of quantum particle physics particulary in connections with both string and $\varepsilon^{\infty}$ theory which were given and studied by El-Naschie $[5,6]$.

Mursaleen and Mohiuddine [8], introduced Fréchet differentiation of nonlinear operators between intuitionistic fuzzy normed spaces. The investigation of nonlinear phenomenons in nature leads to many challenging problems such as; surface waves of fluids, behavior of plastic materials, spreading of bacteria in epidemics, scattering processes of elementary particles and interactions of quantum fields in quantum field theory. In [9] Mursaleen and Mohiuddine studied the ideal convergence of double sequences in probabilistic normed spaces. In 2011, Mursaleen and Lohani introduced and studied statistical limit superior and limit inferior in probabilistic normed spaces [8].

In this paper, we develop classical techniques of nonlinear functional analysis of operators along with Fréchet derivative in Menger probabilistic normed spaces.

\section{Preliminaries}

For reader's convenience, in this section we briefly recall some concepts and results from probabilistic metric spaces theory used in the paper.

Definition 2.1. A function $F: \mathbf{R} \rightarrow[0,1]$ is called a distribution function if it is nondecreasing and left-continuous, with $\sup _{t \in \mathbf{R}} F(t)=1$ and 
inf $f_{t \in \mathbf{R}} F(t)=0$.

The class of all distribution functions $F$ with $F(0)=0$ is denoted by $D_{+} \cdot \varepsilon_{0}$ is the element of $D_{+}$defined by

$$
\varepsilon_{0}= \begin{cases}1, & t>0 \\ 0, & t \leq 0\end{cases}
$$

Definition 2.2. A binary operation $*:[0,1] \times[0,1] \rightarrow[0,1]$ is said to be a t-norm, if it satisfies the following conditions:

(1) $*$ is commutative and associative,

(2) $*$ is continuous,

(3) $a * 1=a$ for all $a \in[0,1]$,

(4) $a * b \leq c * d$ whenever $a \leq c$ and $b \leq d$ for each $a, b, c, d \in[0,1]$.

Definition 2.3 (4). . Let $X$ be a real vector space, $F$ a mapping from $X$ to $D_{+}$(for any $x \in X, F(x)$ is denoted by $F_{x}$ ) and $*$ a t-norm. The triple $(X, F, *)$ is called a Menger probabilistic normed space (briefly Menger PNspace) if the following conditions are satisfied:

1) $F_{x}(0)=0$, for all $x \in X$;

2) $F_{x}=\varepsilon_{0}$ iff $x=\theta$;

3) $F_{\alpha x}(t)=F_{x}\left(\frac{t}{|\alpha|}\right)$ for all $\alpha \in \mathbf{R}, \alpha \neq 0$ and $x \in X$;

4) $F_{x+y}\left(t_{1}+t_{2}\right) \geq F_{x}\left(t_{1}\right) * F_{y}\left(t_{2}\right)$, for all $x, y \in X$ and $t_{1}, t_{2}>0$.

Definition 2.4. Let $(X, F, *)$ be a Menger $P N$-space and let $\left\{x_{n}\right\}$ be a sequence in $X$. Then $\left\{x_{n}\right\}$ is said to be convergent if there exists $x \in X$ such that

$$
\lim _{n \rightarrow \infty} F_{x_{n}-x}(t)=1
$$

for all $t>0$. In this case $x$ is called the limit of $\left\{x_{n}\right\}$.

Definition 2.5. The sequence $\left\{x_{n}\right\}$ in Menger $P N$-space $(X, F, *)$ is called Cauchy if for each $\epsilon>0$ and $t>0$, there exists some $n_{0}$ such that $F_{x_{n}-x_{m}}(t)>1-\epsilon$ for all $m, n \geq n_{0}$.

Clearly, every convergent sequence in Menger PN-space is Cauchy. If each Cauchy sequence is convergent sequence in a Menger $\mathrm{PN}$-space $(X, F, *)$, then $(X, F, *)$ is called Menger probabilistic Banach space (briefly, Menger PB-space). 


\section{Strongly and weakly Menger probabilistic limits}

In this section, we define strongly and weakly Menger probabilistic limits and then we consider their continuity.

Definition 3.1. Let $(X, F, *)$ be a Menger $P N$-space and $U \subset X$. $U$ is said to be MP-open if for each $x \in U$ there exist some $t>0$ and some $\alpha \in(0,1)$ such that $B(x, \alpha, t) \subseteq U$ where $B(x, \alpha, t)=\left\{y: F_{x-y}(t)>1-\alpha\right\}$.

Theorem 3.2. Let $(X, F, *)$ be a Menger $P N$-space with the condition

$$
F_{x}(t)>0 \text { for all } t>0 \text {, implies that } x=0 \text {. }
$$

Let $\|x\|_{\alpha}=\inf \left\{t>0: F_{x}(t) \geq \alpha\right\}$, for each $\alpha \in(0,1)$. Then $\left\{\|\cdot\|_{\alpha}\right.$ : $\alpha \in(0,1)\}$ is an ascending family of norms on $X$. These norms are called $\alpha$-norms on $X$ corresponding to Menger probabilistic norm.

Proof. We only show that:

$$
\begin{gathered}
\|x\|_{\alpha}+\|y\|_{\alpha}=\inf \left\{s: F_{x}(s) \geq \alpha\right\}+\inf \left\{t: F_{y}(t) \geq \alpha\right\}=\inf \{s+t: \\
\left.F_{x}(s) \geq \alpha, F_{y}(t) \geq \alpha\right\}=\inf \left\{s+t: F_{x}(s) * F_{y}(t) \geq \alpha * \alpha\right\} \geq \inf \{s+t: \\
\left.F_{x+y}(s+t) \geq \alpha\right\}=\|x+y\|_{\alpha} .
\end{gathered}
$$

Now, let $0<\alpha_{1}<\alpha_{2}<1$. $\|x\|_{\alpha_{1}}=\inf \left\{t: F_{x}(t) \geq \alpha_{1}\right\}$ and $\|x\|_{\alpha_{2}}=$ inf $\left\{t: F_{x}(t) \geq \alpha_{2}\right\}$. Since $\alpha_{1}<\alpha_{2},\left\{t: F_{x}(t) \geq \alpha_{2}\right\} \subseteq\left\{t: F_{x}(t) \geq \alpha_{1}\right\}$. So $\|x\|_{\alpha_{2}} \geq\|x\|_{\alpha_{1}}$. Thus, we see that $\left\{\|x\|_{\alpha}: \alpha \in(0,1)\right\}$ is an ascending family of norms on $X$.

Definition 3.3. Let $\left\{x_{n}\right\}$ be a sequence in a Menger PN-space $(X, F, *)$. Then

1) It is said to be weakly Menger P-convergent, briefly, WMP-convergent, to $x \in X$ and is denoted by $x_{n} \rightarrow(w m p) x$ iff, for every $\alpha \in(0,1)$ and $\varepsilon>0$, there exists some $n_{0}=n_{0}(\alpha, \varepsilon)$ such that $n \geq n_{0}$ implies $F x_{n}-x(\varepsilon) \geq 1-\alpha$.

2) It is said to be strongly Menger P-convergent, briefly, SMP-convergent, to $x \in X$ and denoted by $x_{n} \rightarrow($ smp) $x$ iff, for every $\alpha \in(0,1)$, there exists some $n_{0}=n_{0}(\alpha)$ such that $n \geq n_{0}$ implies $F_{x_{n}-x}(t) \geq 1-\alpha$, for all $t>0$.

Hence we can derive the definition of the SMP(WMP)-Cauchy sequence and $\mathrm{SMP}(\mathrm{WMP})$-complete Menger PN-space from the above definition as is in the classical cases. 
Proposition 3.4. Let $\left\{x_{n}\right\}$ be a sequence in a Menger PN-space $(X, F, *)$ satisfying the condition (3.1). Then

0 .

1) $x_{n} \rightarrow(w m p) x$ if and only if, for each $\alpha \in(0,1), \lim _{n \rightarrow \infty}\left\|x_{n}-x\right\|_{\alpha}=$

2) $x_{n} \rightarrow(s m p) x$ if and only if, for each $\alpha \in(0,1), \lim _{n \rightarrow \infty}\left\|x_{n}-x\right\|_{\alpha}=0$ uniformly in $\alpha \in(0,1)$.

Proof. 1) Suppose that $x_{n} \rightarrow(w m p) x$. Choose $\alpha \in(0,1)$ and $t>0$. There exists $k \in \mathbf{N}$ such that $F_{x_{n}-x}(t)>1-\alpha$, for all $n \geq k$. It follows that $\left\|x_{n}-x\right\|_{1-\alpha} \rightarrow 0$. Conversely, let $\left\|x_{n}-x\right\|_{\alpha} \rightarrow 0$, for every $\alpha \in(0,1)$. Fix $\alpha \in(0,1)$ and $t>0$. There exists $k \in \mathbf{N}$ such that inf $\left\{r>0: F_{x_{n}-x}(r) \geq\right.$ $1-\alpha\}<t$, for all $n \geq k$. It implies that $F_{x_{n}-x}(t) \geq 1-\alpha$, for all $n \geq k$, i.e., $x_{n} \rightarrow(w m p) x$.

2) Similarly, with the same method we can prove (2).

Theorem 3.5. If a sequence $\left\{x_{n}\right\}$ is SMP-convergent, then it is WMPconvergent to the same limit, but not conversely.

Proof. It is obvious that SMP-convergence implies WMP-convergence. But the converse of this assertion may not be true as the next example shows.

Example 3.6. Let $X$ be a normed space and define

$$
F_{x}(t)= \begin{cases}\frac{t-\|x\|}{t+\|x\|}, & t>\|x\| ; \\ 0, & t \leq\|x\| .\end{cases}
$$

on $X$, and suppose that $a * b=a b$. It is easy to denote that $F$ is Menger probabilistic norm on $X$. We can find the $\alpha$-norm of $F$ since it satisfies the condition (3.1). Thus, $F_{x}(t) \geq \alpha \Leftrightarrow \frac{t-\|x\|}{t+\|x\|} \geq \alpha \Leftrightarrow \frac{1+\alpha}{1-\alpha}\|x\| \leq t$. This shows that $\|x\|_{\alpha}=\inf \left\{t>0: F_{x}(t) \geq \alpha\right\} \leq \frac{1+\alpha}{1-\alpha}\|x\|$. Moreover, $F_{x}\left(\frac{1+\alpha}{1-\alpha}\|x\|\right)=\alpha$, whence $\frac{1+\alpha}{1-\alpha}\|x\| \in\left\{t>0: F_{x}(t) \geq \alpha\right\}$. This means $\|x\|_{\alpha}=\frac{1+\alpha}{1-\alpha}\|x\|$. Let $y \in S_{X}=\{x \in X:\|x\|=1\}$ be fixed and define the sequence $\left\{x_{n}\right\}=\{y / n\}$. We now show that the sequence $\left\{x_{n}\right\}=\{y / n\}$ is WMP-convergent to 0 . This is easy, since for each $\alpha \in(0,1)$,

$$
\left\|x_{n}-0\right\|_{\alpha}=\left(\frac{1+\alpha}{1-\alpha}\right) \frac{\|y\|}{n} \rightarrow 0, \text { as } n \rightarrow \infty .
$$

However, this convergence is not uniform in $\alpha$. Indeed, for given $\varepsilon>0$, $\|x\|_{\alpha}=\left(\frac{1+\alpha}{1-\alpha}\right) \frac{\|y\|}{n}<\varepsilon \Leftrightarrow \frac{1+\alpha}{(1-\alpha) \varepsilon}<n$, whence, we can not find desired $n$. Since $\frac{1+\alpha}{(1-\alpha) \varepsilon} \rightarrow \infty$ as $\alpha \rightarrow 1$. 
Definition 3.7. Let $(X, F, *)$ and $(Y, G, *)$ be two Menger PN-spaces and $f: X \rightarrow Y$ be a mapping.

1) $f$ is called weakly Menger probabilistic continuous at $x_{0} \in X$ if for given $\varepsilon>0$ and $\alpha \in(0,1)$, there exists some $\delta=\delta(\varepsilon, \alpha)>0$ such that, for all $x \in X$

$$
F_{x-x_{0}}(\delta) \geq \alpha \text { implies } G_{f(x)-f\left(x_{0}\right)}(\varepsilon) \geq \alpha .
$$

2) $f$ is called strongly Menger probabilistic continuous at $x_{0} \in X$ if for given $\varepsilon>0$, there exists some $\delta=\delta(\varepsilon)>0$ such that, for all $x \in X$

$$
G_{f(x)-f\left(x_{0}\right)}(\varepsilon) \geq F_{x-x_{0}(\delta)} .
$$

3) Let $f$ be linear. Then $f$ is called weakly Menger probabilistic bounded on $X$ if for every $\alpha \in(0,1)$, there exists some $m_{\alpha}>0$ such that, for all $x \in X$

$$
F_{x}\left(\frac{t}{m_{\alpha}}\right) \geq \alpha \text { implies } G_{f(x)}(t) \geq \alpha, \forall t>0 .
$$

The set of all these operators is denoted by $F^{\prime}(X, Y)$ and it is a vector space.

4) Let $f$ be linear. Then $f$ is called strongly Menger probabilistic bounded on $X$ if for every $\alpha \in(0,1)$, there exists some $M>0$ such that, for all $x \in X$

$$
G_{f(x)}(t) \geq F_{x}\left(\frac{t}{M}\right), \forall t>0 .
$$

The set of all these operators is denoted by $F(X, Y)$ and it is a vector space.

Theorem 3.8. Let $(X, F, *)$ and $(Y, G, *)$ be two Menger $P N$-spaces and $f: X \rightarrow Y$ be a linear mapping. Then, $f$ is strongly (weakly) Menger probabilistic continuous if and only if it is strongly (weakly) Menger probabilistic bounded.

Proof. First we suppose that $f$ is strongly Menger probabilistic bounded. Thus there exists $M>0$ such that

$$
G_{f(x)}(t) \geq F_{x}\left(\frac{t}{M}\right) \quad \forall x \in X, \forall t>0,
$$

so

$$
G_{f(x)-f(0)}(t) \geq F_{x-0}\left(\frac{t}{M}\right),
$$


hence

$$
G_{f(x)-f(0)}(t) \geq F_{x-0}(\delta) \text { where } \delta=\frac{\varepsilon}{M} .
$$

This implies that $f$ is strongly Menger probabilistic continuous at 0 and hence it is strongly Menger probabilistic continuous on $X$. Using strong continuity of $f$ at $x=0$, for $\varepsilon=1$, there exists $\delta>0$ such that

$$
G_{f(x)-f(0)}(1) \geq F_{x-0}(\delta) \quad \forall x \in X .
$$

Suppose that $x \neq 0$ and $t>0$. Putting $u=x / t$ then

$$
\begin{gathered}
G_{f(x)}(t)=G_{t f(u)}(t)=G_{f(u)}(1) \geq F_{u}(\delta)=F_{x / t}(\delta)=F_{x}(t / M) \text { where } \\
M=1 / \delta .
\end{gathered}
$$

So $G_{f(x)}(t) \geq F_{x}(t / M)$. If $x \neq 0$ and $t \leq 0$ then $G_{f(x)}(t)=0=$ $F_{x}(t / M)$. If $x=0$ and $t \in \mathbf{R}$ then $f(0)=0$ and

$$
\begin{aligned}
& G_{0}(t)=F_{0}(t / M)=1 \text { if } t>0, \\
& G_{0}(t)=F_{0}(t / M)=0 \text { if } t \leq 0 .
\end{aligned}
$$

From the above discussion it follows that, $f$ is strongly Menger probabilistic bounded.

Theorem 3.9. Let $(X, F, *)$ be a Menger PN-space satisfying (3.1) and the condition

For $x \neq 0, F_{x}($.$) is continuous on \mathbf{R}$ and strictly increasing on

$$
\left\{t: 0<F_{x}(t)<1\right\} .
$$

Also suppose that $\{\|\|:. \alpha \in(0,1)\}$ be the family of corresponding $\alpha$-norms of $F$ on $X$ defined by $\|x\|_{\alpha}=\inf \left\{t: F_{x}(t) \geq \alpha\right\}, \alpha \in(0,1)$. Then for any increasing (or decreasing) sequence $\left\{\alpha_{n}\right\}$ in $(0,1), \alpha_{n} \rightarrow \alpha$ implies $\|x\|_{\alpha_{n}} \rightarrow\|x\|_{\alpha} \quad \forall x \in X$.

Proof. For $x=0$, it is clear that $\alpha_{n} \rightarrow \alpha \Rightarrow\|x\|_{\alpha_{n}} \rightarrow\|x\|_{\alpha}$. Suppose $x \neq 0$. From Lemma (1.2) [2], for $x \neq 0, \alpha \in(0,1)$ and $t^{\prime}>0$ we have $\|x\|_{\alpha}=t^{\prime}$ iff $F_{x}\left(t^{\prime}\right)=\alpha$. Let $\left\{\alpha_{n}\right\}$ be an increasing sequence in $(0,1)$ such that $\alpha_{n} \rightarrow \alpha \in(0,1)$. Let $\|X\|_{\alpha_{n}}=t_{n}$ and $\|x\|_{\alpha}=t$. Then

$$
F_{x}\left(t_{n}\right)=\alpha_{n} \text { and } F_{x}(t)=\alpha \text {. }
$$


We know the sequence $\left\{t_{n}\right\}$ is an increasing sequence of real numbers and it is bounded above by $t$. Hence $\left\{t_{n}\right\}$ is convergent. Thus

$$
F_{x}\left(\lim _{n \rightarrow \infty} t_{n}\right)=\alpha .
$$

From (3.3) and (3.4) we have $F_{x}\left(\lim _{n \rightarrow \infty}\left(t_{n}\right)\right)=F_{x}(t)$ so by (3.2) we have $\lim _{n \rightarrow \infty} t_{n}=t$. Therefore, $\lim _{n \rightarrow \infty}\|x\|_{\alpha_{n}}=\|x\|_{\alpha}$. Similarly, if $\left\{\alpha_{n}\right\}$ is a decreasing sequence, we can prove the theorem.

Theorem 3.10. Let $(X, F, *)$ and $(Y, G, *)$ be two Menger $P N$-spaces satisfying (3.1) and (3.2) and suppose that $f: X \rightarrow Y$ be a linear mapping. Then

1) $f$ is weakly Menger probabilistic bounded if and only if it is bounded with respect to $\alpha$-norms of $F$ and $G$, for each $\alpha \in(0,1)$.

2) $f$ is strongly Menger probabilistic bounded if and only if it is uniformly bounded with respect to $\alpha$-norms of $F$ and $G$.

Proof. 1) First we suppose that $f$ is weakly Menger probabilistic bounded. Thus for every $\alpha \in(0,1)$ there exists $m_{\alpha}>0$ such that for all $x \in X$ and $t \in \mathbf{R}$ we have $F_{x}\left(\frac{t}{m_{\alpha}}\right) \geq \alpha$ and then $G_{f(x)}(t) \geq \alpha$. Hence $\sup \left\{\beta \in(0,1):\left\|m_{\alpha} x\right\|_{\beta}^{1} \leq t\right\} \geq \alpha \Rightarrow \sup \left\{\beta \in(0,1):\|f(x)\|_{\beta}^{2} \leq t\right\} \geq \alpha$. Now we show that,

$$
\sup \left\{\beta \in(0,1):\left\|m_{\alpha} x\right\|_{\beta}^{1} \leq t\right\} \geq \alpha \Leftrightarrow\left\|m_{\alpha} x\right\|_{\alpha}^{1} \leq t .
$$

If $x=0$ then the relation is obvious. Suppose $x \neq 0$.

$$
\text { Now if } \sup \left\{\beta \in(0,1):\left\|m_{\alpha} x\right\|_{\beta}^{1} \leq t\right\}>\alpha \text { then }\left\|m_{\alpha} x\right\|_{\alpha}^{1} \leq t .
$$

If $\sup \left\{\beta \in(0,1):\left\|m_{\alpha} x\right\|_{\beta}^{1} \leq t\right\}=\alpha$, then there exists an increasing sequence $\left\{\alpha_{n}\right\}$ such that $\alpha_{n} \rightarrow \alpha$ and $\left\|m_{\alpha} x\right\|_{\alpha_{n}}^{1} \leq t$. Then by Theorem (3.9) we have

$$
\left\|m_{\alpha} x\right\|_{\alpha}^{1} \leq t
$$

Thus from (3.5) and (3.6) we get, $\sup \left\{\beta \in(0,1):\left\|m_{\alpha} x\right\|_{\beta} \leq t\right\} \geq \alpha \Rightarrow$ $\left\|m_{\alpha} x\right\|_{\alpha}^{1} \leq t$. Next we suppose that

$$
\left\|m_{\alpha} x\right\|_{\alpha}^{1} \leq t
$$

If $\left\|m_{\alpha} x\right\|_{\alpha}^{1}<t$ then $F_{m_{\alpha} x}(t) \geq \alpha$. So

$$
\sup \left\{\beta \in(0,1):\left\|M_{\alpha} x\right\|_{\beta}^{1} \leq t\right\} \geq \alpha .
$$


If $\left\|m_{\alpha} x\right\|_{\alpha}^{1}=t$, then there exists a decreasing sequence $\left\{s_{n}\right\}$ in $\mathbf{R}$ such that $s_{n} \rightarrow t$ and $F_{m_{\alpha} x}\left(s_{n}\right) \geq \alpha \Rightarrow F_{m_{\alpha} x}\left(\lim _{n \rightarrow \infty} s_{n}\right) \geq \alpha$ so by (3.2) we get $F_{m_{\alpha} x}(t) \geq \alpha$. Hence

$$
\sup \left\{\beta \in(0,1):\left\|m_{\alpha} x\right\|_{\beta}^{1} \leq t\right\} \geq \alpha .
$$

From (3.8) and (3.9) it follows that,

$$
\left\|m_{\alpha} x\right\|_{\alpha} \leq t \Rightarrow \sup \left\{\beta \in(0,1):\left\|m_{\alpha} x\right\|_{\beta}^{1} \leq t\right\} \geq \alpha .
$$

Hence from (3.7) and (3.10) we have

$$
\sup \left\{\beta \in(0,1):\left\|m_{\alpha} x\right\|_{\beta} \leq t\right\} \geq \alpha \Leftrightarrow\left\|m_{\alpha} x\right\|_{\alpha}^{1} \leq t .
$$

In a similar way we can show that,

$$
\sup \left\{\beta \in(0,1):\|f(x)\|_{\beta}^{2} \leq t\right\} \geq \alpha \Leftrightarrow\|f(x)\|_{\alpha}^{2} \leq t .
$$

Therefore from (3.11) and (3.12) we have $F_{m_{\alpha} x}(t) \geq \alpha \Rightarrow G_{f(x)}(t) \geq \alpha$ then $\left\|m_{\alpha} x\right\|_{\alpha}^{1} \leq t \Rightarrow\|f(x)\|_{\alpha}^{2} \leq t$. This implies that $\|f(x)\|_{\alpha}^{2} \leq m_{\alpha}\|x\|_{\alpha}^{1}$.

Conversely suppose that for every $\alpha \in(0,1)$, there exists $m_{\alpha}>0$ such that $\|f(x)\|_{\alpha}^{2} \leq m_{\alpha}\|x\|_{\alpha}^{1}$. Then for $x \neq 0$, inf $\left\{s: F_{m_{\alpha} x}(s) \geq \alpha\right\} \leq t \Rightarrow$ $\inf \left\{s: G_{f(x)}(s) \geq \alpha\right\} \leq t$.

In a similar way as above we can show that $\inf \left\{s: F_{m_{\alpha} x}(s) \geq \alpha\right\} \leq$ $t \Leftrightarrow F_{m_{\alpha} x}(t) \geq \alpha$ and $\inf \left\{s: G_{f(x)}(s) \geq \alpha\right\} \leq t \Leftrightarrow G_{f(x)}(t) \geq \alpha$. Thus we have $F_{x}\left(\frac{t}{m_{\alpha}}\right) \geq \alpha \Rightarrow G_{f(x)}(t) \geq \alpha$. If $x \neq 0, t \leq 0$ and $x=0, t>0$ then the above relation is obvious. Hence the Theorem follows.

2) Let $\|.\|_{\alpha}^{1}$ and $\|.\|_{\alpha}^{2}$ be the $\alpha$-norms of $F$ and $G$ respectively. First we suppose that $f$ is strongly Menger probabilistic bounded. Thus, there exists $M>0$ such that for all $x \in X$ and $s \in \mathbf{R}$ we have $G_{f(x}(s) \geq F_{x}\left(\frac{s}{M}\right)$. So

$$
G_{f(x)}(s) \geq F_{M x}(s) .
$$

$$
\begin{gathered}
\text { Now }\|M x\|_{\alpha}^{1}<t \Rightarrow \inf \left\{s: F_{M x}(s) \geq \alpha\right\}<t \\
\Rightarrow \exists s_{0}<t \quad \text { such that } \quad F_{M x}\left(s_{0}\right) \geq \alpha \\
\Rightarrow \exists s_{0}<t \quad \text { such that } \quad G_{f(x)}\left(s_{0}\right) \geq \alpha \\
\Rightarrow\|f(x)\|_{\alpha}^{2} \leq s_{0}<t .
\end{gathered}
$$


Hence $\|f(x)\|_{\alpha}^{2} \leq\|M x\|_{\alpha}^{1}=M\|x\|_{\alpha}^{1}$. This implies that $T$ is uniformly bounded with respect to $\alpha$-norms.

Conversely suppose that, there exists $M>0$ such that $\|f(x)\|_{\alpha}^{2} \leq$ $M\|x\|_{\alpha}^{1}$ holds for all $\alpha \in(0,1)$ and $x \in X$. Now $r<F_{M x}(s)$

$$
\begin{gathered}
\Rightarrow r<\sup \left\{\alpha \in(0,1):\|M x\|_{\alpha}^{1} \leq s\right\} \\
\Rightarrow \exists \alpha_{0} \in(0,1) \quad \text { such that } \quad r<\alpha_{0} \quad \text { and }\|M x\|_{\alpha_{0}}^{1} \leq s \\
G_{f(x)}(s) \geq F_{M x}(s)=F_{x}\left(\frac{s}{M}\right) .
\end{gathered}
$$

So $f$ is strongly Menger probabilistic bounded and hence the theorem follows.

Definition 3.11. Let $(X, F, *)$ and $(Y, G, *)$ be two Menger $P N$-spaces satisfying (3.1) and (3.2) and $f: X \rightarrow Y$ be a linear mapping. Then,

1) $L$ is said to be strong Menger probabilistic limit of $f$ at some $x_{0} \in X$ iff given $\varepsilon>0$, there exists some $\delta=\delta(\varepsilon)>0$ such that

$$
G_{f(x)-L}(\varepsilon) \geq F_{x-x_{0}}(\delta) .
$$

In this case, we use the symbol $s f-\lim _{x \rightarrow x_{0}} f(x)=L$.

2) $L$ is said to be weak Menger probabilistic limit of $f$ at some $x_{0} \in X$ iff for given $\varepsilon>0$ and $\alpha \in(0,1)$, there exists some $\delta=\delta(\varepsilon, \alpha)>0$ such that

$$
F_{x-x_{0}}(\delta) \geq \alpha \quad \text { implies } \quad G_{f(x)-L}(\varepsilon) \geq \alpha .
$$

In this case, we use the symbol $w f-\lim _{x \rightarrow x_{0}} f(x)=L$.

Proposition 3.12. $s f$-lim implies $w f$-lim but not conversely, in general. Further, $s f-l i m=w f-l i m$ whenever $s f-l i m$ exists.

Proof. The implication easily can be seen from the definition. Now let $s f-\lim _{x \rightarrow x_{0}} f(x)=L$ and $w f-\lim _{x \rightarrow x_{0}} f(x)=L_{1}$. Then

$$
G_{L_{1}-L}(2 t)=G_{L_{1}-f(x)+f(x)-L}(2 t) \geq \min \left\{G_{f(x)-L_{1}}(t), G_{f(x)-L}(t)\right\}
$$

for every $t>0$. Hence we get $G_{L_{1}-L}(2 t)=1$, by passing the $s f$ and $w f$-limits as $F_{x-x_{0}}(t) \rightarrow 1$. So $L_{1}=L$ from the definition of Menger probabilistic norm.

Now let us show the implication may be strict. 
Example 3.13. Let $X=Y=\mathbf{R}$,

$$
F_{x}(t)= \begin{cases}\frac{t}{t+|x|}, & t>0 \\ 0, & t \leq 0\end{cases}
$$

and

$$
G_{x}(t)= \begin{cases}1, & t>|x| \\ 0, & t \leq|x|\end{cases}
$$

Also we define $a * b=a b$. Consider the function $f(x)=x$ from $(\mathbf{R}, F, *)$ onto $(\mathbf{R}, G, *)$. Then $w f-\lim _{x \rightarrow 0} f(x)=0$. Indeed let $\varepsilon>0$ and $\alpha \in(0,1)$ be given. $G_{f(x)-L}(\varepsilon)=G_{x}(\varepsilon) \geq \alpha$ implies $\varepsilon>|x|$. So if we take $\delta=\frac{\alpha \varepsilon}{1-\alpha}>$ 0 then

$$
F_{x-x_{0}}(\delta)=F_{x}(\delta)=\frac{\alpha \varepsilon}{1-\alpha} \cdot \frac{1-\alpha}{\alpha \varepsilon+(1-\alpha)|x|} \geq \frac{\alpha \varepsilon}{\alpha \varepsilon+(1-\alpha) \varepsilon}=\alpha .
$$

However, $s f-\lim _{x \rightarrow 0} f(x)$ does not exist. Because, for $|x|=\varepsilon$, there is no $\delta>0$ satisfying the condition

$$
G_{x}(\varepsilon)=0 \geq F_{x}(\delta)=\frac{\delta}{\delta+|x|}=\frac{\delta}{\delta+\varepsilon} .
$$

By above definitions we can redefine the strongly and weakly Menger probabilistic continuity of mappings between Menger probabilistic normed spaces.

Definition 3.14. Let $(X, F, *)$ and $(Y, G, *)$ be two Menger $P N$-spaces and $f: X \rightarrow Y$ be a mapping.

1) $f$ is said to be strongly Menger probabilistic continuous at some $x_{0} \in X$ iff $s f-\lim _{x \rightarrow x_{0}} f(x)=f\left(x_{0}\right)$.

2) $f$ is said to be weakly Menger probabilistic continuous at some $x_{0} \in$ $X$ iff $w f-\lim _{x \rightarrow x_{0}} f(x)=f\left(x_{0}\right)$.

Proposition 3.15. Let $(X, F, *)$ and $(Y, G, *)$ be two Menger PN-spaces satisfying (3.1) and $f: X \rightarrow Y$ be a mapping. Then

1) $w f-\lim _{x \rightarrow x_{0}} f(x)=L$ if and only if, for each $\alpha \in(0,1), \lim _{\left\|x-x_{0}\right\|_{\alpha}^{1} \rightarrow 0} \| f(x)-$ $L \|_{\alpha}^{2}=0$.

2) $s f-\lim _{x \rightarrow x_{0}} f(x)=L$ if and only if $\lim _{\left\|x-x_{0}\right\|_{\alpha}^{1} \rightarrow 0}\|f(x)-L\|_{\alpha}^{2}=0$ uniformly in $\alpha$.

Where $\|.\|_{\alpha}^{1}$ and $\|.\|_{\alpha}^{2}$ are $\alpha$-norms of the Menger probabilistic norms $F$ and $G$, respectively. 
Proof. Since first one is easier than the second, let us prove only the second part. Suppose $s f-\lim _{x \rightarrow x_{0}} f(x)=L$, that is, given $\varepsilon>0$, there exists some $\delta=\delta(\varepsilon)>0$ such that for every $x \in X$,

$$
G_{f(x)-L}(\varepsilon) \geq F_{x-x_{0}}(\delta) .
$$

Now, for each $\alpha \in(0,1)$, if

$$
\left\|x-x_{0}\right\|_{\alpha}^{1}=\inf \left\{t>0: F_{x-x_{0}}(\delta) \geq \alpha\right\} \leq \delta
$$

then $F_{x-x_{0}}(\delta) \geq \alpha$. Hence $G_{f(x)-L}(\varepsilon) \geq \alpha$ by the hypothesis, so that $\|f(x)-L\|_{\alpha}^{2} \leq \varepsilon$. Since $\delta$ does not depend on $\alpha$ this shows that

$$
\lim _{\left\|x-x_{0}\right\|_{\alpha}^{1} \rightarrow 0}\|f(x)-L\|_{\alpha}^{2}=0 \quad \text { uniformly } \quad \text { in } \quad \alpha .
$$

Conversely, let $\lim _{\left\|x-x_{0}\right\|_{\alpha}^{1} \rightarrow 0}\|f(x)-L\|_{\alpha}^{2}=0$ uniformly in $\alpha \in(0,1)$. For given $\varepsilon>0$, there exists some $\delta=\delta(\varepsilon)>0$ such that for every $x \in X$,

$$
\left\|x-x_{0}\right\|_{\alpha}^{1} \leq \delta \text { implies }\|f(x)-L\|_{\alpha}^{2} \leq \varepsilon .
$$

Consider some $\lambda<F_{x-x_{0}}(\delta)$. Since

$$
F_{x-x_{0}}(\delta)=\sup \left\{\alpha \in(0,1):\left\|x-x_{0}\right\|_{\alpha}^{1} \leq \delta\right\},
$$

there exists some $\alpha_{0} \in(0,1)$ such that $\lambda<\alpha_{0}$ and $\left\|x-x_{0}\right\|_{\alpha}^{1} \leq \delta$. Hence $\|f(x)-L\|_{\alpha}^{2} \leq \varepsilon$ by the hypothesis. So, we can get $G_{f(x)-L}(\varepsilon) \geq F_{x-x_{0}}(\delta)$.

\section{Differentiation of nonlinear operators in Menger proba- bilistic normed spaces}

In this section we define the Menger probabilistic Fréchet differentiation and then we investigate this definition in Menger probabilistic normed spaces.

Definition 4.1. Let $(X, F, *)$ and $(Y, G, *)$ be two Menger PN-spaces, $U \subseteq$ $X$ be a Menger probabilistic open subset and $f: U \rightarrow Y$ ba a mapping, probably nonlinear. Then $f$ is called strong(weak) Menger probabilistic Fréchet differentiation at $x_{0} \in U$ if there exists a strongly(weakly) Menger probabilistic bounded linear operator $T$ from $(X, F, *)$ to $(Y, G, *)$ such that

$$
\lim _{F_{h}(t) \rightarrow 1} G_{\frac{f\left(x_{0}+h\right)-f\left(x_{0}\right)-T h}{1-F_{h}(t)}}(t)=1(s f(w f)), \quad \forall t>0 .
$$


The operator $T$ is called strong(weak) Menger probabilistic, or briefly, sf(wf)-Fréchet, derivative of $f$ at $x_{0}$ and is denoted by $d_{s f(w f)} f\left[x_{0}\right] . f$ is called $s f(w f)$-Fréchet differentiable on $U$ if it is $s f(w f)$-Fréchet differentiable at every point of $U$. In this case, $x \rightarrow d_{s f(w f)} f[x]$ is a function from $U$ to $F(X, Y)\left(F^{\prime}(X, Y)\right)$ which we denote it by $d_{s f(w f)} f$.

Theorem 4.2. A strongly(weakly) Menger probabilistic bounded linear operator $A$ is $s f(w f)$-Fréchet differentiable at every point $x_{0}$ and $d_{s f(w f)} A\left[x_{0}\right]=$ A.

Proof. This is obvious since $G_{\frac{A\left(x_{0}+h\right)-A\left(x_{0}\right)-A h}{1-F_{h}(t)}}(t)=G_{0}(t)=1, \quad \forall t>0$.

Proposition 4.3. If $f$ is $s f(w f)$-Fréchet differentiable at $x_{0} \in U$, then it is strongly (weakly) Menger probabilistic continuous at $x_{0}$.

Proof. This can be easily proved as in the classical case by considering following inequalities and by definitions. For every $t>0$

$$
\begin{gathered}
G_{f(x)-f\left(x_{0}\right)}(t)=G_{f(x)-f\left(x_{0}\right)-T h+T h}(t) \geq \min \left\{G_{f(x)-f\left(x_{0}\right)-T h}(t(1-\right. \\
\left.\left.\left.F_{h}(t)\right)\right), G_{T h}\left(t F_{h}(t)\right)\right\}=\min \left\{G_{\frac{f(x)-f\left(x_{0}\right)-T h}{1-F_{h}(t)}}(t), G_{\frac{T h}{F_{h}(t)}}(t)\right\}
\end{gathered}
$$

where $T=d_{s f(w f)} f\left[x_{0}\right]$ and remember that it is strongly(weakly) Menger probabilistic continuous.

Theorem 4.4. Let $(X, F, *)$ and $(Y, G, *)$ be two Menger PN-spaces, $U \subseteq$ $X$ be a Menger probabilistic open subset and $f: U \rightarrow Y$ ba a mapping. If $f$ is sf-Fréchet differentiable at some $x_{0} \in U$ then it is wf-Fréchet differentiable at $x_{0}$ with the same derivative but not conversely.

Proof. The proof is directly comes from the Proposition (3.12). For the counterexample let us consider the following example.

Example 4.5. Let $X=Y=c_{0}$, the Banach space of all sequences converging to 0 with the supnorm $\|x\|_{\infty}=\sup _{n}\left|x_{n}\right|$, where $x=\left\{x_{n}\right\}_{n=1}^{\infty}$. Define the functions

$$
F_{x}(t)= \begin{cases}\frac{t^{2}}{t^{2}+2\|x\|_{\infty}}, & t>0 \\ 0, & t \leq 0\end{cases}
$$


and

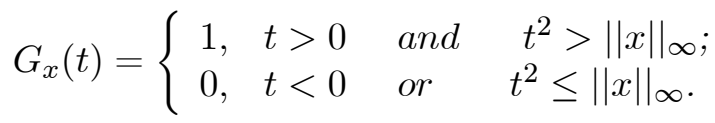

Also suppose that $a * b=a b$. It is not hard to show that $F$ and $G$ are Menger probabilistic norms on $c_{0}$. Consider the shift operator $S(x)=$ $S\left(\left\{x_{1}, x_{2}, \ldots\right\}\right)=\left\{0, x_{1}, x_{2}, \ldots\right\}$ on $c_{0}$. Let us show that linear operator $S$ is weakly bounded hence is weakly continuous from $\left(c_{0}, F, *\right)$ into $\left(c_{0}, G, *\right)$. Let $\alpha \in(0,1)$ be given. For every $x \in c_{0}$,

$$
F_{m_{\alpha} x}(t) \geq \alpha \Rightarrow \frac{t^{2}}{t^{2}+2 m_{\alpha}\|x\|_{\infty}} \geq \alpha \Leftrightarrow t^{2} \geq \frac{2 \alpha m_{\alpha}\|x\|_{\infty}}{1-\alpha}=\frac{2 \alpha m_{\alpha}\|S(x)\|_{\infty}}{1-\alpha} .
$$

Hence if we take $m_{\alpha}>\frac{1-\alpha}{2 \alpha}$,

$$
F_{m_{\alpha} x}(t) \geq \alpha \Rightarrow t^{2}>\|S(x)\|_{\infty} \Leftrightarrow G_{S(x)}(t)=1>\alpha .
$$

This proves $S$ is weakly bounded. However, for $x \in c_{0}$ such that $t^{2} \leq$ $\|x\|_{\infty}, G_{S(x)}(t)=0$, hence we can not find any $M>0$ such that

$$
\forall x \in c_{0} \text { and } \forall t \in \mathbf{R}, G_{S(x)}(t) \geq F_{x}(t / M) .
$$

This implies that $S$ can not be strongly continuous. Since $S$ is linear we get from Theorem (4.2) that $S=d_{w f} S[x]$ for every $x \in c_{0}$, while $d_{s f} S[x]$ does not exist.

Theorem 4.6. Let $(X, F, *)$ and $(Y, G, *)$ be two Menger PN-spaces satisfying (3.1), $U \subseteq X$ be a Menger probabilistic open subset and $f: U \rightarrow Y$ ba a mapping. Then,

1) $f$ is sf-Fréchet differentiable at some $x_{0} \in U$ with $T=d_{s f} f\left[x_{0}\right]$ if and only if, for each $\alpha \in(0,1)$,

$$
\lim _{\|h\|_{\alpha}^{1} \rightarrow 0} \frac{\left\|f\left(x_{0}+h\right)-f\left(x_{0}\right)-T h\right\|_{\alpha}^{G}}{\|h\|_{\alpha}^{F}}=0
$$

2) $f$ is wf-Fréchet differentiable at some $x_{0} \in U$ with $T=d_{w f} f\left[x_{0}\right]$ if and only if

$$
\lim _{\|h\|_{\alpha}^{1} \rightarrow 0} \frac{\left\|f\left(x_{0}+h\right)-f\left(x_{0}\right)-T h\right\|_{\alpha}^{G}}{\|h\|_{\alpha}^{F}}=0 \text { uniformly in } \alpha,
$$

where $\|.\|_{\alpha}^{F}$ and $\|.\|_{\alpha}^{G}$ are $\alpha$-norms of the Menger PN-spaces, respectively. 
Proof. The proof of the Theorem directly comes from Proposition (3.15).

Theorem 4.7. Let $(X, F, *),(Y, G, *)$ and $(Z, H, *)$ Menger PN-spaces, $U \subseteq X$ and $V \subseteq Y$ be a Menger probabilistic open subsets.

1) Let $f, g: U \rightarrow Y$ be a mapping and $d_{s f(w f)} f\left[x_{0}\right], d_{s f(w f)} g\left[x_{0}\right]$ exist. Then $d_{s f(w f)}(f+g)\left[x_{0}\right]$ exists and $d_{s f(w f)}(f+g)\left[x_{0}\right]=d_{s f(w f)} f\left[x_{0}\right]+$ $d_{s f(w f)} g\left[x_{0}\right]$.

2) Suppose that $f: U \rightarrow Y$ and $g: V \rightarrow Z$ are such that $g \circ f: U \rightarrow Z$ is defined, $d_{s f(w f)} f\left[x_{0}\right]$ and $d_{s f(w f)} g\left[f\left(x_{0}\right)\right]$ exist. Then $d_{s f(w f)}(g \circ f)\left[x_{0}\right]$ exists and

$$
d_{s f(w f)}(g \circ f)\left[x_{0}\right]=d_{s f(w f)} g\left[f\left(x_{0}\right)\right] \circ d_{s f(w f)} f\left[x_{0}\right] .
$$

Proof. Since proof of the first part is elementary, let us prove the second. Suppose $F_{h}(t) \neq 1$. Let us say $\Lambda\left(x_{0}, h\right)=g\left(f\left(x_{0}+h\right)\right)-g\left(f\left(x_{0}\right)\right), A=$ $d_{s f(w f)} g\left[f\left(x_{0}\right)\right]$ and $B=d_{s f(w f)} f\left[x_{0}\right]$. So, $g\left(f\left(x_{0}\right)+f\left(x_{0}+h\right)-f\left(x_{0}\right)\right)-$ $g\left(f\left(x_{0}\right)\right)=\Lambda\left(x_{0}, h\right)$ and for every $t>0$,

$$
\frac{H_{\Lambda\left(x_{0}, h\right)-\left(A\left(f\left(x_{0}+h\right)-f\left(x_{0}\right)\right)\right)}}{1-G_{f\left(x_{0}+h\right)-f\left(x_{0}\right)^{(t)}}}(t) \rightarrow 1(s f(w f)),
$$

as $G_{\left(f\left(x_{0}+h\right)-f\left(x_{0}\right)\right.}(t) \rightarrow 1$. But

$$
H_{\frac{\Lambda\left(x_{0}, h\right)-\left(A\left(f\left(x_{0}+h\right)-f\left(x_{0}\right)\right)\right)}{1-F_{h}(t)}}(t) \rightarrow 1(s f(w f))
$$

as $F_{h}(t) \rightarrow 1$, since $f$ is strongly (weakly) continuous at $x_{0}$. Let us show this assertion for strongly continuity, at least. Given $\varepsilon>0$, there exists some $\delta_{1}(\varepsilon)>0$ such that

$$
\frac{H_{\Lambda\left(x_{0}, h\right)-\left(A\left(f\left(x_{0}+h\right)-f\left(x_{0}\right)\right)\right)}}{1-G_{f\left(x_{0}+h\right)-f\left(x_{0}\right)^{(t)}}}(\varepsilon) \geq G_{f\left(x_{0}+h\right)-f\left(x_{0}\right)}\left(\delta_{1}\right) .
$$

We can also find another $\delta=\delta\left(\delta_{1}\right)>0$ such that

$$
G_{f\left(x_{0}+h\right)-f\left(x_{0}\right)}\left(\delta_{1}\right) \geq F_{h}(\delta),
$$

by the strongly continuity of $f$. So, this proves the assertion. Now, remember that

$$
G_{\frac{f\left(x_{0}+h\right)-f\left(x_{0}\right)-B h}{1-F_{h}(t)}}(t) \rightarrow 1(s f(w f))
$$

as $F_{h}(t) \rightarrow 1$. Hence 


$$
\begin{gathered}
\frac{\Lambda\left(x_{0}, h\right)-A(B h)}{1-F_{h}(t)}=\frac{\Lambda\left(x_{0}, h\right)-A\left\{f\left(x_{0}+h\right)-f\left(x_{0}\right)-B h-\left(f\left(x_{0}+h\right)-f\left(x_{0}\right)\right)\right\}}{1-F_{h}(t)}= \\
\frac{\Lambda\left(x_{0}, h\right)-A\left(f\left(x_{0}+h\right)-f\left(x_{0}\right)\right)}{1-F_{h}(t)}-A\left(\frac{f\left(x_{0}+h\right)-f\left(x_{0}\right)-B h}{1-F_{h}(t)}\right) .
\end{gathered}
$$

If $P=\frac{\Lambda\left(x_{0}, h\right)-A\left(f\left(x_{0}+h\right)-f\left(x_{0}\right)\right)}{1-F_{h}(t)}$ and $Q=A\left(\frac{f\left(x_{0}+h\right)-f\left(x_{0}\right)-B h}{1-F_{h}(t)}\right)$ then

$$
H_{\frac{\Lambda\left(x_{0}, h\right)-A(B h)}{1-F_{h}(t)}}(t)=H_{P+Q}(t) \geq \min \left\{H_{P}(t / 2), H_{Q}(t / 2)\right\} \rightarrow 1(s f(w f))
$$

since each term in $\min$ tends to $1(s f(w f))$ as $F_{h}(t) \rightarrow 1$.

Theorem 4.8. Let $(X, F, *)$ and $(Y, G, *)$ be two Menger PB-spaces satisfying (3.1) and (3.2), $U \subseteq X$ be a convex Menger probabilistic open subset and $f: U \rightarrow Y$ be a mapping such that $d_{s f} f\left[x_{0}\right]$ exists for each $x_{0} \in U$. Further, suppose that for each $\alpha \in(0,1)$

$$
\sup \left\{\left\|d_{s f} f[x]\right\|_{\alpha}: x \in U\right\}=m_{\alpha}<\infty
$$

where

$$
\left\|d_{s f} f[x]\right\|_{\alpha}=\sup \left\{\left\|d_{s f} f[x]\right\|_{\beta}^{*}: \beta \leq \alpha\right\}
$$

and

$$
\left\|d_{s f} f[x]\right\|_{\beta}^{*}=\sup \left\{\left\|\frac{d_{s f} f[x] v \|_{\beta}^{G}}{\|v\|_{\beta}^{F}}\right\|:\|v\|_{\beta}^{F} \leq 1, v \neq 0, v \in X\right\} .
$$

Then for every $x_{1}, x_{2} \in U$, there exists some $M>0$ (independent of $\alpha$ ) such that

$$
\left\|f\left(x_{1}\right)-f\left(x_{2}\right)\right\|_{\alpha}^{G} \leq M\left\|x_{1}-x_{2}\right\|_{\beta}^{F}
$$

for each $\alpha \in(0,1)$, where $\|.\|_{\alpha}^{F}$ and $\|.\|_{\beta}^{G}$ are $\alpha$-norms of the Menger PNspaces $F$ and $G$, respectively.

Proof. By the Theorem (4.6), $f$ is Fréchet differentiable as a mapping between Banach spaces $\left(X,\|\cdot\|_{\alpha}^{F}\right)$ and $\left(Y,\|.\|_{\alpha}^{G}\right)$, for each $\alpha \in(0,1)$. First of all we will show that there is $M>0$ (independent of $\alpha$ ) such that $\sup _{\alpha \in(0,1)} m_{\alpha} \leq M$. Since $d_{s f} f[x]$ is strongly Menger probabilistic bounded, there exists $M_{1}>0$ (independent of $\alpha$ ) such that

$$
\left\|d_{s f} f[x] v\right\|_{\alpha}^{G} \leq M_{1}\|v\|_{\alpha}^{F}, v \in X, \alpha \in(0,1) .
$$

By Theorem (3.10) This means $\left\|d_{s f} f[x]\right\|_{\alpha}^{*} \leq M$. Since $x \in U$ is arbitrary this implies 


$$
\sup \left\{\left\|d_{s f} f[x]\right\|_{\alpha}: x \in U\right\} \leq M, \text { for each } \alpha \in(0,1) .
$$

This is desired result.

On the other hand, the classical mean-value theorem in Banach spaces tell us by this result that

$$
\left\|f\left(x_{1}\right)-f\left(x_{2}\right)\right\|_{\alpha}^{G} \leq m_{\alpha}\left\|x_{1}-x_{2}\right\|_{\alpha}^{F},
$$

for each $\alpha \in(0,1)$ []. So immediately we can write

$$
\left\|f\left(x_{1}\right)-f\left(x_{2}\right)\right\|_{\alpha}^{G} \leq M\left\|x_{1}-x_{2}\right\|_{\alpha}^{F}
$$

\section{Compact operators between Menger probabilistic normed spaces}

In this section, at first we define the concept of $\operatorname{smp}(w m p)$-compact and then we consider some results.

Definition 5.1. A subset $B$ in a Menger probabilistic normed space $(X, F, *)$ is called $\operatorname{smp}(w m p)$-compact if each sequence of elements of $B$ has a $\operatorname{smp}(w m p)$ convergent subsequence.

Obviously, every smp-compact set is wmp-compact, but not viceversa.

Example 5.2. Let $X$ be a normed space and define

$$
F_{x}(t)= \begin{cases}\frac{t-\|x\|}{t+\|x\|}, & t>\|x\| ; \\ 0, & t \leq\|x\| .\end{cases}
$$

and $G_{x}(t)=1-F_{x}(t)$ on $X$. It is easy to denote that $F, G$ are Menger probabilistic normed spaces on $X$ by defining $a * b=a b$. We can find the $\alpha$-norms of $F, G$ since it satisfies the condition (3.1). Thus,

$$
\begin{aligned}
& F_{x}(t) \geq \alpha \Leftrightarrow \frac{t-\|x\|}{t+\|x\|} \geq \alpha \Leftrightarrow \frac{1+\alpha}{1-\alpha}\|x\| \leq t \text { and } \\
& G_{x}(t) \leq 1-\alpha \Leftrightarrow F_{x}(t) \geq \alpha \Leftrightarrow \frac{1+\alpha}{1-\alpha}\|x\| \leq t .
\end{aligned}
$$

This shows that $\|x\|_{\alpha}=\inf \left\{t>0: F_{x}(t) \geq \alpha, G_{x}(t) \leq 1-\alpha\right\} \leq$ $\frac{1+\alpha}{1-\alpha}\|x\|$. Moreover, 


$$
F_{x}\left(\frac{1+\alpha}{1-\alpha}\|x\|\right)=\alpha \text { and } G_{x}\left(\frac{1+\alpha}{1-\alpha}\|x\|\right)=1-\alpha,
$$

whence,

$$
\frac{1+\alpha}{1-\alpha}\|x\| \in\left\{t>0: F_{x}(t) \geq \alpha, G_{x}(t) \leq 1-\alpha\right\} .
$$

This means that $\|x\|_{\alpha}=\frac{1+\alpha}{1-\alpha}\|x\|$. Let $y \in\{x \in X:\|x\|=1\}$ be fixed and define $\left\{x_{n}\right\}=\left\{\frac{y}{n}\right\}$. We now show that the sequence $\left\{x_{n}\right\}=\left\{\frac{y}{n}\right\}$ is wmp-convergent to 0 . This is easy since for each $\alpha \in(0,1)$,

$$
\left\|x_{n}-x\right\|_{\alpha}=\left(\frac{1+\alpha}{1-\alpha}\right) \frac{\|y\|}{n} \rightarrow 0 \text {, as } n \rightarrow \infty .
$$

However, this convergence is not uniform in $\alpha$. Indeed for given $\varepsilon>0$,

$$
\|x\|_{\alpha}=\left(\frac{1+\alpha}{1-\alpha}\right) \frac{\mid y \|}{n}<\varepsilon \Leftrightarrow \frac{1+\alpha}{(1-\alpha) \varepsilon}<n,
$$

whence, we can not find desired $n_{0}$ since $\frac{1+\alpha}{(1-\alpha) \varepsilon} \rightarrow \infty$ as $\alpha \rightarrow 1$.

Definition 5.3. The $\operatorname{smp}(w m p)$-closure of a subset $B$ in a Menger PNspace $(X, F, *)$ is denoted by $B^{-s}\left(B^{-w}\right)$ and defined by the set of all $x \in X$ such that there exists a sequence $\left\{x_{n}\right\}$ in $B$ such that $x_{n} \rightarrow \operatorname{smp}(w m p) x$. We say that $B$ is $\operatorname{smp}\left(\right.$ wmp)-closed whenever $B^{-s}\left(B^{w}\right)=B$.

It is easy to see that $B^{-s} \subseteq B^{-w}$. Let us present an example showing that this inclusion may be strict.

Example 5.4. Take $X=\mathbf{C}$, in the example (5.2). Then the unit sphere $B_{X}=\{x \in X:|x| \leq 1\}$ is wmp-compact in $(X, F, *)$. However, it is not smp-compact. Indeed the sequence $\left\{\frac{1}{n}\right\}$ can not have a smp-convergent subsequence as is shown in the last part of the example (5.2).

Definition 5.5. Let $(X, F, *)$ and $(Y, G, *)$ be two Menger PN-spaces and $f: X \rightarrow Y$ ba a mapping. Then, $f$ is called $\operatorname{smp}(w m p)$-compact if for every Menger probabilistic bounded subset $B$ of $X$ the subset $f(B)$ is relatively $\operatorname{smp}(w m p)$-compact, that is, $\operatorname{smp}(w m p)$-closure of $f(B)$ is $\operatorname{smp}(w m p)$-compact.

Theorem 5.6. Let $(X, F, *)$ and $(Y, G, *)$ be two Menger $P N$-spaces and $f: X \rightarrow Y$ ba a mapping. Then $f$ is $\operatorname{smp}(w m p)$-compact if and only if it maps every Menger probabilistic bounded sequence $\left\{x_{n}\right\}$ in $X$ onto a sequence $\left\{f\left(x_{n}\right)\right\}$ in $Y$ which has a $\operatorname{smp}(w m p)$-convergent subsequence. 
Proof. The proof has a similar way with the classical counterpart.

Remark 5.7. Of course, every smp-compact operator is wmp-compact, but not vice-versa.

Theorem 5.8. Let $(X, F, *)$ and $(Y, G, *)$ be two Menger PN-spaces satisfying the condition (3.1) and $f: X \rightarrow Y$ be a mapping. Suppose that $f$ is wmp-compact. Then, $f$ is an ordinary compact operator from the normed $\operatorname{space}\left(X,\|\cdot\|_{\alpha}\right)$ into $\left(Y,\|\cdot\|_{\alpha}\right)$ for each $\alpha \in(0,1)$.

Proof. Let $\alpha \in(0,1)$ be arbitrary and pick some arbitrary bounded sequence $\left\{x_{n}\right\} \subset\left(X,\|.\|_{\alpha}\right)$ and say $K=\sup _{n}\left\|x_{n}\right\|_{\alpha}<\infty$. So, $F_{x_{n}}(K) \geq \alpha$ for each $n=1,2, \ldots$, by the definition of $\|.\|_{\alpha}$. Thus, $\left\{x_{n}\right\}$ is a Menger probabilistic bounded sequence in $X$. There exists a wmp-convergent subsequence $\left\{f\left(x_{n_{k}}\right)\right\}$ of $\left\{f\left(x_{n}\right)\right\}$ in $Y$ by the hypothesis. Hence $\left\{f\left(x_{n_{k}}\right)\right\}$ is convergent in the normed space $\left(Y,\|.\|_{\alpha}\right)$ by the proposition (3.12).

Theorem 5.9. Let $(X, F, *)$ and $(Y, G, *)$ be two Menger PN-spaces satisfying the condition (3.1) and $f: X \rightarrow Y$ be a mapping. Then every $\operatorname{smp}$ (wmp)-compact linear operator $f: X \rightarrow Y$ is $\operatorname{smp}(w m p)$-continuous.

Proof. The proof can be easily away carried out in the classical analogue of the result.

Theorem 5.10. Let $(X, F, *)$ and $(Y, G, *)$ be two wf-complete Menger $P N$-spaces and $f: X \rightarrow Y$ be a nonlinear wf-compact operator. Suppose that, for some $x_{0} \in X, d_{w f} f\left[x_{0}\right]=A$ exists. Then the linear operator $A$ is also wf-compact.

Proof. Let $\left\{x_{n}\right\} \subset(X, F, *)$ be an arbitrary Mengar probabilistic bounded sequence. This means, there exist some $t_{0}>0$ and $r \in(0,1)$ such that $F_{x_{n}}\left(t_{0}\right) \geq 1-r$, for every positive integer $n$. Consider the sequence $\left\{x_{0}+x_{n}\right\}_{n=1}^{\infty}$ and let us show it is Menger probabilistic bounded. If we take $t^{\prime}=2 t_{0}$ and $r^{\prime}=1-\min \left\{1-r, F_{x_{0}}\left(t_{0}\right)\right\}$, then

$$
F_{x_{0}+x_{n}}\left(t^{\prime}\right) \geq \min \left\{F_{x_{n}}\left(t_{0}\right), F_{x_{0}}\left(t_{0}\right)\right\} \geq \min \left\{1-r, F_{x_{0}}\left(t_{0}\right)\right\}=1-r^{\prime},
$$

for every positive integer $n$, whence, the result comes. Hence, by the hypothesis there exists a subsequence $\left\{x_{0}+x_{n_{k}}\right\}$ of $\left\{x_{0}+x_{n}\right\}$ such that $\left\{f\left(x_{0}+x_{n_{k}}\right)\right\}$ is wf-convergent, hence wf-Cauchy in $(Y, G, *)$ as well. There is no loss of generality to write $x_{n}$ for $x_{n_{k}}$ again. Let 


$$
\Lambda\left(x_{0}, h\right)=f\left(x_{0}+h\right)-f\left(x_{0}\right)-A h .
$$

Then

$$
A x_{n}-A x_{m}=\Lambda\left(x_{0}, x_{m}\right)-\Lambda\left(x_{0}, x_{n}\right)+f\left(x_{0}+x_{n}\right)-f\left(x_{0}+x_{m}\right) .
$$

Since $A=d_{w f} f\left[x_{0}\right]$ for every $\varepsilon>0$ and $\alpha \in(0,1)$, we can find some $\delta_{m}(\varepsilon, \alpha) \geq t_{0}>0$ such that

$$
G_{\Lambda\left(x_{0}, x_{m}\right)}\left(\frac{\varepsilon}{r}\left(1-F_{x_{m}}\left(\delta_{m}\right)\right)\right) \geq 1-\alpha \text {, if } F_{x_{m}}\left(\delta_{m}\right) \geq 1-\alpha .
$$

Now, $\delta \geq t_{0}$ means $F_{x_{m}}\left(\delta_{m}\right) \geq F_{x_{m}}\left(t_{0}\right) \geq 1-r \Leftrightarrow 1-F_{x_{m}}\left(\delta_{m}\right) \leq r$, and so this implies

$$
\begin{gathered}
G_{\Lambda\left(x_{0}, x_{n}\right)}(\varepsilon)=G_{\Lambda\left(x_{0}, x_{m}\right)}\left(\frac{\varepsilon}{r} r\right) \geq G_{\Lambda\left(x_{0}, x_{m}\right)}\left(\frac{\varepsilon}{r}\left(1-F_{x_{m}}\left(\delta_{m}\right)\right)\right) \geq 1-\alpha, \text { if } \\
F_{x_{m}}\left(\delta_{m}\right) \geq 1-\alpha .
\end{gathered}
$$

Further, with the same way we can find some $\delta_{n}(\varepsilon, \alpha) \geq t_{0}>0$

$$
G_{\Lambda\left(x_{0}, x_{n}\right)}(\varepsilon) \geq 1-\alpha \text {, if } F_{x_{n}}\left(\delta_{n}\right) \geq 1-\alpha .
$$

On the other hand,

$$
G_{A x_{n}-A x_{m}}(3 t) \geq \min \left\{G_{\Lambda\left(x_{0}, x_{n}\right)}(t), G_{\Lambda\left(x_{0}, x_{m}\right)}(t), G_{f\left(x_{0}+x_{n}\right)-f\left(x_{0}+x_{m}\right)}(t)\right\}
$$

and, since $\left\{f\left(x_{0}+x_{n}\right)\right\}$ is wf-Cauchy, for every $\alpha \in(0,1)$ and $\varepsilon>0$, there exists some $n_{0}=n_{0}(\alpha, \varepsilon)$ such that $n, m \geq n_{0}$ implies $G_{\left(f\left(x_{0}+x_{n}\right)-f\left(x_{0}+x_{m}\right)\right.}(\varepsilon) \geq$ $1-\alpha$. Consequently, we obtain

$$
G_{A x_{n}-A x_{m}}(3 \varepsilon) \geq \min \{1-\alpha, 1-\alpha, 1-\alpha\}=1-\alpha
$$

for $n, m \geq n_{0}$. This completes the proof.

It is clear that this result is true for sf-compact operators as well.

\section{References}

[1] C. Alsina, B. Schweizer and A. Sklar, On the definition of a probabilistic normed space, Aequationes Math. 46, pp. 91-98, (1993).

[2] T. Bag and S. K. Samanta, Finite dimensional fuzzy normed linear spaces, The journal of Fuzzy Mathematics, 11, pp. 687-705, (2003). 
[3] B. Buffoni and J. Toland, Analytic theory of global bifurcation, Princeton Oxford: Princeton University Press; (2003).

[4] S. S. Chang, Y. J. Cho and S. M. Kang, Probabilistic metric spaces and nonlinear operator theory, Sichuan University Press, Chengdu, (1994).

[5] M. S. El Naschie, On the uncertainly of Cantorian geometry and twoslit experiment, Choas, Solitions and Fractals, 9, pp. 517-529, (1998).

[6] M. S. El Naschie, On the unification of heterotic strings, $M$ theory and $\varepsilon^{\infty}$ theory, Choas, Solitions and Fractals, 11, pp. 2397-2408, (2000).

[7] K. Mengar, Statistical metrics, Proc. Nat. Acad. Sci. 28, pp. 535-537, (1942).

[8] M. Mursaleen and Q. M. Danish Lohani, Statistical limit superior and limit inferior in probabilistic normed spaces, Filomat, 25 (3), pp. 55-67, (2011).

[9] M. Mursaleen and S. A. Mohiuddine, On ideal convergence of double sequences in probabilistic normed spaces, Math. Reports, 12 (64) (4), pp. 359-371, (2010).

[10] M. Mursaleen and S. A. Mohiuddine, Nonlinear operators between intuituinistic fuzzy normed spaces and Frechet derivative, Chaos, solitions and Fractals, 42 (2), pp. 1010-1015, (2009).

[11] A. N. Šerstnev, On the notion of a random normed space, Dokl. Akad. Nauk. 149, pp. 280-283, (1963).

\author{
N. Eghbali \\ Department of Mathematics and Applications, \\ Faculty of Mathematical Sciences, \\ University of Mohaghegh Ardabili, \\ 56199-11367, Ardabil, \\ Iran \\ e-mail : eghbali@uma.ac.ir
}

